\title{
EDITORIAL
}

\section{A brief account of viral vectors and their promise for gene} \section{therapy}

\author{
Gene Therapy (2017) 24, 1-2; doi:10.1038/gt.2016.71
}

The development of viral vectors for gene therapy has had an interesting and somewhat tumultuous history but remains the most important avenue tor treatment of a large number of diseases both heritable and acquired. The demonstration by the Varmus/Bishop lab in the mid-1970s that $\gamma$-retroviruses can naturally acquire cellular genes provided a strong argument for the use of viruses to deliver novel genetic material to cells. Within a few years, experimental confirmation of this concept was presented by several labs using recombinants of both integrating (retrovirus) and mostly non-integrating DNA viruses (SV40, BPV (bovine papilloma virus), vaccinia and HSV (herpes simplex virus)). Retroviruses were considered attractive because integration would assure stable gene delivery and passed along in daughter cells. The mystery of retroviral genome integration requiring conversion of the viral RNA genome into DNA had earlier been solved by Howard Temin and David Baltimore with the discovery of reverse transcriptase. Some years later, advances in molecular cloning provided the means to more readily and accurately engineer retroviral vectors for acceptance and expression of therapeutic genes. Other improvements along the way included the development of packaging cell lines by Richard Mulligan and many others to produce replication-defective retroviral vectors and implement pseudotyping with VSV-G glycoprotein to expand vector tropism. Luigi Naldini and Inder Verma were among the first to create lentiviral vectors that added the capability of gene delivery to non-dividing cells. These vectors remain in use today to engineer hematopoietic stem cells to correct recessive monogenetic diseases affecting, for example, T-cell development and function, and to transduce a variety of tissues in vivo including liver and brain. Lentiviral vectors are having an important role in immuno-oncology through their use in engineering tumor-specific cytotoxic T cells. Also under development are stem-cell applications combining retroviral transduction with control of transgene expression using microRNA recognition sites embedded into transgene constructs. Retroviral vectors remain the best systems for ex vivo gene therapy despite potential problems related to insertional activation of oncogenes or inactivation of tumor suppressor functions.

Since these first vectors were developed, various vector systems have enjoyed rapid development and in the early days, transfer of $\beta$-galactosidase using, for example, adenoviral vectors was a favorite marker to demonstrate gene transfer-the blue period of the gene therapy field. Later on, imaging of fluorescent markers or luciferase in intact animals evolved as informative means of documenting gene transfer. There is now a wealth of clinical experience with numerous vector types that include primarily vaccinia, measles, vesicular stomatitis virus (VSV), polio, reovirus, adenovirus, lentivirus, $\mathrm{\gamma}$-retrovirus, adeno-associated virus (AAV) and herpes simplex virus (HSV). Vaccinia, measles, VSV, polio, reovirus, adeno and HSV vectors have settled into a role as vaccines and cancer therapeutics, lenti- and $\gamma$-retroviral vectors are predominantly used for transduction of transplantable cells, and AAV is a currently favored vehicle for direct gene delivery to specific tissues such as liver. Together, these vectors have enabled treatments primarily of tumors and rare metabolic diseases such as melanoma and hemophilia, respectively. The first gene therapies have been approved (UniQure's Glybera, Amgen's Imlygic and Glaxosmithkline's StremvelisTM) and others are likely to be approved within 5 years to treat retinal degeneration, such as leber congenital amaurosis and sickle cell anemia as examples. Despite these advances, there remain significant hurdles that include antiviral responses that block initial vector delivery, prevent repeat dosing, and typically cause the eventual loss of transgene expression. Cell-mediated vector delivery may hold promise to overcome these hurdles but has yet to gain mainstream attention.

The development of AAV vectors, primarily the early pioneering work of Ken Berns, Barrie Carter, Nicholas Muzyczka and Jude Samulski, has blossomed as perhaps the most powerful in vivo gene therapy tool. Systemic delivery is made possible by forced genetic selection or engineering of viral capsids, the work of Arun Srivastava and David Strayer among many others, and by selection of natural strains of virus from primates promulgated primarily by the work of James Wilson and Guangping Gao. It may be possible to avoid problems related to immune surveillance through genetic engineering of AAV capsids, thereby opening the door to more effective delivery to the target tissue. Further insights into the biology of AAV uncoating and gene expression are emerging and that should increase efficiency of gene transfer and reduce immune-cell activation. Although AAV currently has limited packaging potential $(4.7 \mathrm{~kb})$, removing it from consideration for larger promoter-gene payloads, it is not pathogenic and preclinical vector distribution studies, indicate the great promise of these vectors for the treatment of a variety of diseases.

Viral vectors have emerged as a potentially potent approach to the treatment of cancer by selective lytic virus replication in tumors. Arming of oncolytic vectors with immuno-modulatory genes is also under intense investigation as a means to enhance virus-induced antitumor immunity. Tumors create an environment that discourages both innate and acquired immunity and manipulation of the tumor microenvironment using viral vectors armed with genes to unblock the local immune response is in the early stages of development. Antibodies that foil checkpoint molecules causing cytotoxic T-cell exhaustion are already in preclinical and early clinical evaluation in combination with viral vectors using either systemic administration of those antibodies or expression of transgenes encoding these antibodies from oncolytic viral genomes. Vector delivery itself is a central issue in the use of viruses to treat cancer. Most people believe that systemic delivery would be preferred if possible, but currently intratumoral administration is the gold standard. To make systemic delivery feasible, methods for stealthing infection to overcome pre-existing antiviral immunity and prevention of potentionally dangerous inflammatory responses will be needed. The need for systemic vector delivery may be blunted by vectors that on local delivery, rapidly induce robust antitumor immunity. Antitumor immunity could prove effective against metastatic disease.

A problem related to the induction of antitumor immunity concerns the low number of tumor-specific-epitopes in most cancer types and these neoantigens are generally the result of mutations related to expression of cancer driving genes. These include mutations that cause oncogene activation (for example, 
ras), loss of tumor suppressor gene products (for example, $p 53$ ), loss of apoptotic mechanisms (for example, $B c / 2$ ), loss of cell cycle control genes (for example, p16) and mutations affecting growth factor receptors or their downstream pathways (for example, EGFRvIII). Some tumors also re-express early developmental or embryonic products (for example, CEA). In addition, the immune system is designed to prevent auto-immunity through several mechanisms, which include the activity of regulatory T cells and prevention of self-antigen presentation by dendritic cells. Nevertheless, the use of oncolytic viruses to treat solid tumors by direct intratumoral inoculation has led to accumulating evidence for a phenomenon referred to as epitope spreading. In essence, the ability of the virus to induce antiviral responses locally attracts lymphocytes and macrophages that are highly activated and likely to recognize tumor antigens released as a result of virolysis, thereby greatly enhancing tumor epitope availability for activation of T-helper cells. The presence of tumor-specific immunity has been demonstrated by tumor rejection in tumor challenge experiments in the absence of virus infection, suggesting that oncolytic viruses can increase the likelihood that antitumor immunity will develop. The combination of checkpoint molecular inhibition with viral oncolytic activity may prove to be an exciting strategy in the treatment of cancer.

Perhaps the most interesting new areas in the field relate to the treatment of central nervous system diseases, many of which have unknown etiology and likely involve complex pathways with multiple subtle changes that cause neurodegeneration, brain aging, epilepsy, addiction or mental illness. There are two significant challenges at hand. The first is that neurodegenerative diseases affect the brain broadly although symptoms arise that point to specific brain regions such as the substantia nigra in Parkinson's disease, the striatum in Huntington's disease and the hippocampus in epilepsy. Effective treatments will require in many cases, high capacity vectors, such as HSV and broad-based delivery to the brain that is currently difficult using viral vectors due to the blood-brain barrier. Although progress in brain penetration by systemic delivery of AAV, for example, is moving forward, there remain a vast number of neurons that are uninfected. In addition, some diseases will require delivery to non-neuronal brain cell types such as astrocytes, for which transduction is even less well developed. The second challenge will be to tailor gene transfer and expression in a manner to target specific pathways in selected cell types. It will take a considerable amount of work to engineer highly selective transductional, transcriptional and post-transcriptional targeting systems combined with multigene transfer and appropriate expression timed to correct defective interactive pathways. In addition, neuromodulation will come into play with drug regulation to silence or activate neuronal activities using specifically designed, pharmacologically responsive ion channels and G-protein coupled receptors. Neuromodulation represents an important new frontier in gene therapy that can be implemented in sensory nerves for the treatment of chronic pain and in the brain for conditions such as tremor and epilepsy.

The future of the gene therapy field is likely to take a turn toward introducing methods for gene correction using targeted homologous recombination in vivo. This is especially true from dominant monogenic diseases. Many metabolic diseases, such as cystic fibrosis and Duchenne muscular dystrophy, have proven to be very difficult targets for current gene therapy strategies and efficient viral vector-mediated delivery of the machinery to correct such mutant genes in vivo will be a landmark achievement. Gene editing tools may also be applied to inactivation or correction of defective genes in embryos that will cause disease during development or in adult tissues. The use of these advanced methods will depend on the assurance of safety and specificity that are yet to be fully realized. No doubt this will engender hot debate on both practical and ethical grounds.

The science of gene therapy is enjoying a renaissance after an initial roller-coaster trajectory followed by a period of high negativity. The heart of this field still largely relies on viral vector gene transfer systems that have evolved considerably. Still more advanced platforms and vehicle delivery systems are required to enable appropriate level and duration of gene expression in targeted cell types. There is great hope for this area of therapeutics and it is no small wonder that a slew of gene therapy start-up companies has emerged that have received many millions of dollars in private and public investments. Many nonprofit foundations have also made huge contributions to the development of gene therapy applications. The cost of preclinical and clinical gene therapy research is enormous and will take these combined communities to support the further realization of this type of medical practice. The reality is that gene therapy holds great promise for diseases that are well beyond the development of standard pharmaceuticals and the fulfillment of this promise remains the driver for the excitement in this field.

\section{CONFLICT OF INTEREST}

M Finer is a Founder and CEO of Oncorus inc., Founder of Switch Bio Inc., and a Director of MPM Capitol, Cambridge, MA. J Glorioso is a Founder of Oncorus Inc., Switch Bio Inc. and Nuvovec srl. Italy.

M Finer ${ }^{1}$ and J Glorioso ${ }^{2}$
'Oncorus Inc., Cambridge, MA, USA and
${ }^{2}$ Department of Microbiology and Molecular Genetics, University of
Pittsburgh School of Medicine, Pittsburgh, PA, USA
E-mail: glorioso@pitt.edu

$\mathrm{M}$ Finer $^{1}$ and $\mathrm{J}$ Glorioso $^{2}$ (1)

(1)

E-mail: glorioso@pitt.edu 